\title{
Dilatonic dyon black hole solutions in the model with two Abelian gauge fields
}

\author{
Medeu Abishev" ${ }^{1,2,4, \star}$, Dmitry Ivashchuk ${ }^{3,4}$,Kuantay Boshkayev,2, , and Algys Malybayev,2, \\ ${ }^{1}$ IETP, \\ ${ }^{2}$ Physical and Technical Faculty, Al-Farabi Kazakh National University, al'-Farabi avenue, 71, Almaty 050040, \\ Kazakhstan \\ ${ }^{3}$ Center for Gravitation and Fundamental Metrology, VNIIMS, Ozyornaya St., 46, Moscow 119361, Russia \\ ${ }^{4}$ Institute of Gravitation and Cosmology, RUDN University, Miklukho-Maklaya St.,6, Moscow 117198, Russia
}

\begin{abstract}
Dilatonic black hole dyon-like solutions in the gravitational $4 d$ model with a scalar field, two 2-forms, two dilatonic coupling constants $\lambda_{i} \neq 0, i=1,2$, obeying $\lambda_{1} \neq-\lambda_{2}$ and sign parameter $\varepsilon= \pm 1$ for scalar field kinetic term are considered. Here $\varepsilon=-1$ corresponds to ghost scalar field. These solutions are defined up to solutions of two master equations for two moduli functions, when $\lambda_{i}^{2} \neq 1 / 2$ for $\varepsilon=-1$. A set of bounds on gravitational mass and scalar charge are presented by using a certain conjecture on parameters of solutions, when $1+2 \lambda_{i}^{2} \varepsilon>0, i=1,2$.
\end{abstract}

\section{Introduction}

Here we give a brief extension of our previous work [1] devoted to dilatonic dyon black hole solutions. We consider a subclass of dilatonic black hole solutions with electric and magnetic charges $Q_{1}$ and $Q_{2}$, respectively in $4 d$ model with metric $g$, scalar field $\varphi$, two 2-forms $F^{(1)}$ and $F^{(2)}$, corresponding to two dilatonic coupling constants $\lambda_{1}$ and $\lambda_{2}$, respectively. For coinciding dilatonic couplings $\lambda_{1}=\lambda_{2}=\lambda$ we get a trivial non-composite generalisation of dilatonic dyon black hole solutions in the model with one 2-form which was considered in ref. [1]. The dilatonic scalar field may be either an ordinary one or a phantom (or ghost) one.

Here we present lower bounds on gravitational mass $M$ and scalar charge $Q_{\varphi}$. As in ref. [1] this problem is solved here up to a conjecture, which states one to one (smooth) correspondence between the pair $\left(Q_{1}^{2}, Q_{2}^{2}\right)$, where $Q_{1}$ is electric charge and $Q_{2}$ is magnetic charge, and the pair of positive parameters $\left(P_{1}, P_{2}\right)$, which appear in decomposition of moduli functions at large distances. This conjecture is believed to be valid for all $\lambda_{i} \neq 0$ in the case of ordinary scalar field and for $0<\lambda_{i}^{2}<1 / 2$ for the case of phantom scalar field (in both cases the inequality $\lambda_{1} \neq-\lambda_{2}$ is assumed).

\footnotetext{
^e-mail: abishevme@gmail.com
} 


\section{Black hole dyon solutions}

Let us consider a model governed by the action

$$
S=\frac{1}{16 \pi G} \int d^{4} x \sqrt{|g|}\left\{R[g]-\varepsilon(\partial \varphi)^{2}-\sum_{i=1}^{2} \frac{1}{2} e^{2 \lambda_{i} \varphi}\left(F^{(i)}\right)^{2}\right\},
$$

where $g=g_{\mu \nu}(x) d x^{\mu} \otimes d x^{\nu}$ is metric, $\varphi$ is the scalar field, $F^{(i)}=d A^{(i)}=\frac{1}{2} F_{\mu \nu}^{(i)} d x^{\mu} \wedge d x^{\nu}$ is the 2-form with $A^{(i)}=A_{\mu}^{(i)} d x^{\mu}, \varepsilon= \pm 1, G$ is the gravitational constant, $(\partial \varphi)^{2}=g^{\mu \nu} \partial_{\mu} \varphi \partial_{\nu} \varphi,\left(F^{(i)}\right)^{2}=F_{\mu \nu}^{(i)} F^{(i) \mu \nu}$, $i=1,2$. Here $\lambda_{1}, \lambda_{2} \neq 0$ are coupling constants obeying $\lambda_{1} \neq-\lambda_{2}$ and $|g|=\left|\operatorname{det}\left(g_{\mu \nu}\right)\right|$. We put $\lambda_{i}^{2} \neq 1 / 2, i=1,2$, for $\varepsilon=-1$.

We consider a family of dyonic-like black hole solutions to the field equations corresponding to the action (2.1) which are defined on the manifold

$$
\mathcal{M}=(2 \mu,+\infty) \times S^{2} \times \mathbb{R}
$$

and have the following form

$$
\begin{aligned}
& d s^{2}=g_{\mu \nu} d x^{\mu} d x^{\nu}=H_{1}^{h_{1}} H_{2}^{h_{2}}\left\{-H_{1}^{-2 h_{1}} H_{2}^{-2 h_{2}}\left(1-\frac{2 \mu}{R}\right) d t^{2}\right. \\
& \left.+\frac{d R^{2}}{1-\frac{2 \mu}{R}}+R^{2} d \Omega_{2}^{2}\right\} \\
& \exp (\varphi)=H_{1}^{h_{1} \lambda_{1} \varepsilon} H_{2}^{-h_{2} \lambda_{2} \varepsilon} \text {, } \\
& F^{(1)}=\frac{Q_{1}}{R^{2}} H_{1}^{-2} H_{2}^{-A_{12}} d t \wedge d R, \\
& F^{(2)}=Q_{2} \tau \text {. }
\end{aligned}
$$

Here $Q_{1}$ and $Q_{2}$ are (colored) charges - electric and magnetic, respectively, $\mu>0$ is the extremality parameter, $d \Omega_{2}^{2}=d \theta^{2}+\sin ^{2} \theta d \phi^{2}$ is the canonical metric on the unit sphere $S^{2}(0<\theta<\pi, 0<\phi<2 \pi)$, $\tau=\sin \theta d \theta \wedge d \phi$ is the standard volume form on $S^{2}$,

$$
h_{i}=K_{i}^{-1}, \quad K_{i}=\frac{1}{2}+\varepsilon \lambda_{i}^{2}
$$

$i=1,2$, and

$$
A_{12}=\left(1-2 \lambda_{1} \lambda_{2} \varepsilon\right) h_{2}
$$

Functions $H_{s}>0$ obey the equations

$$
R^{2} \frac{d}{d R}\left(R^{2} \frac{\left(1-\frac{2 \mu}{R}\right)}{H_{s}} \frac{d H_{s}}{d R}\right)=-K_{s} Q_{s}^{2} \prod_{l=1,2} H_{l}^{-A_{s l}}
$$

with the following boundary conditions imposed

$$
H_{s} \rightarrow H_{s 0}>0
$$

for $R \rightarrow 2 \mu$, and

$$
H_{s} \rightarrow 1
$$

for $R \rightarrow+\infty, s=1,2$. 
In (2.9) we denote

$$
\left(A_{s s^{\prime}}\right)=\left(\begin{array}{cc}
2 & A_{12} \\
A_{21} & 2
\end{array}\right)
$$

where $A_{12}$ is defined in (2.8) and

$$
A_{21}=\left(1-2 \lambda_{1} \lambda_{2} \varepsilon\right) h_{1} .
$$

These solutions may be obtained just by using general formulae for non-extremal (intersecting) black brane solutions from [2]. The composite analogs of the solutions with one 2-form and $\lambda_{1}=\lambda_{2}$ were presented in ref. [1].

The first boundary condition (2.10) guarantees (up to a possible additional demand on analicity of $H_{s}(R)$ in the vicinity of $R=2 \mu$ ) the existence of (regular) horizon at $R=2 \mu$ for the metric (2.3). The second condition (2.11) ensures an asymptotical (for $R \rightarrow+\infty$ ) flatness of the metric.

\section{Bounds on mass and scalar charge}

For ADM gravitational mass we get from (2.3)

$$
G M=\mu+\frac{1}{2}\left(h_{1} P_{1}+h_{2} P_{2}\right),
$$

where the parameters $P_{s}$ appear in asymptotical relations $H_{s}=1+P_{s} / R+o(1 / R)$, as $R \rightarrow+\infty$. The scalar charge just follows from (2.4)

$$
Q_{\varphi}=\varepsilon\left(\lambda_{1} h_{1} P_{1}-\lambda_{2} h_{2} P_{2}\right)
$$

Here we outline the following hypothesis, which is supported by certain numerical calculations $[1,3]$. For $h_{1}=h_{2}$ this conjecture was proposed in ref. [1].

Conjecture. For any $h_{1}>0, h_{2}>0, \varepsilon= \pm 1, Q_{1} \neq 0, Q_{2} \neq 0$ and $\mu>0$ : (A) the moduli functions $H_{s}(R)$, which obey (2.9), (2.10) and (2.11), are uniquely defined and hence the parameters $P_{1}, P_{2}$, the gravitational mass $M$ and the scalar charge $Q_{\varphi}$ are uniquely defined too; $(B)$ the parameters $P_{1}$, $P_{2}$ are positive and the functions $P_{1}=P_{1}\left(Q_{1}^{2}, Q_{2}^{2}\right), P_{2}=P_{2}\left(Q_{1}^{2}, Q_{2}^{2}\right)$ define a diffeomorphism of $\mathbb{R}_{+}^{2}$ $\left(\mathbb{R}_{+}=\{x \mid x>0\}\right) ;(C)$ in the limiting case we have: (i) for $Q_{2}^{2} \rightarrow+0: P_{1} \rightarrow-\mu+\sqrt{\mu^{2}+K_{1} Q_{1}^{2}}$, $P_{2} \rightarrow+0$ and (ii) for $Q_{1}^{2} \rightarrow+0: P_{1} \rightarrow+0, P_{2} \rightarrow-\mu+\sqrt{\mu^{2}+K_{2} Q_{2}^{2}}$.

The conjecture could be readily verified for the case $\varepsilon=1, \lambda_{1} \lambda_{2}=1 / 2$. Another integrable case $\varepsilon=1, \lambda_{1}=\lambda_{2}=\lambda, \lambda^{2}=3 / 2$ is more involved [3].

The conjecture implies the following proposition.

Proposition. For $h_{s}>0, Q_{s} \neq 0, \lambda_{s} \neq 0(s=1,2)$ and $\lambda_{1}+\lambda_{2} \neq 0$ we have the following bounds on the gravitational mass $M$ and the scalar charge $Q_{\varphi}$ which are valid for all $\mu>0$ :

$$
\begin{array}{r}
\frac{1}{2} \sqrt{h_{\text {min }}\left(Q_{1}^{2}+Q_{2}^{2}\right)}<G M, \\
\left|Q_{\varphi}\right|<|\lambda|_{\max } \sqrt{h_{\text {min }}\left(Q_{1}^{2}+Q_{2}^{2}\right),}
\end{array}
$$

for $\varepsilon=+1\left(0<h_{s}<2\right)$, and

$$
\begin{array}{r}
\sqrt{\frac{1}{2}\left(Q_{1}^{2}+Q_{2}^{2}\right)}<G M, \\
\left|Q_{\varphi}\right|<|\lambda|_{\max } \sqrt{h_{\text {max }}\left(Q_{1}^{2}+Q_{2}^{2}\right)},
\end{array}
$$


for $\varepsilon=-1\left(h_{s}>2\right)$. Here $h_{\min }=\min \left(h_{1}, h_{2}\right), h_{\max }=\max \left(h_{1}, h_{2}\right)$, and $|\lambda|_{\max }=\max \left(|\lambda|_{1},|\lambda|_{2}\right)$; $h_{\text {min }}=\left(\frac{1}{2}+|\lambda|_{\text {max }}^{2}\right)^{-1}$ for $\varepsilon=+1$ and $h_{\text {max }}=\left(\frac{1}{2}-|\lambda|_{\text {max }}^{2}\right)^{-1}$ for $\varepsilon=-1$.

In ref. [1] Proposition was proved for the case $\lambda_{1}=\lambda_{2}$. In this case the bound (3.3) is coinciding (up to notations) with that from ref. [4], which was proved there by using certain spinor techniques.

\section{Conclusions}

Here a family of non-extremal black hole dyon-like solutions in a $4 \mathrm{~d}$ gravitational model with a scalar field and two Abelian vector fields is considered. The scalar field is either ordinary $(\varepsilon=+1)$ or phantom one $(\varepsilon=-1)$. The model contain two dilatonic coupling constants $\lambda_{s} \neq 0, s=1,2$, obeying $\lambda_{1} \neq-\lambda_{2}$.

The solutions are defined up to two moduli functions $H_{1}(R)$ and $H_{2}(R)$, which obey two differential equations of second order with boundary conditions imposed. For $\varepsilon=+1$ these equations are integrable for four cases, corresponding to Lie algebras $A_{1}+A_{1}, A_{2}, B_{2}=C_{2}$ and $G_{2}$. In the first case $\left(A_{1}+A_{1}\right)$ we have $\lambda_{1} \lambda_{2}=1 / 2$, while in the second one $\left(A_{2}\right)$ we get $\lambda_{1}=\lambda_{2}=\lambda$ and $\lambda^{2}=3 / 2$.

Here we have presented lower bounds on the gravitational mass and upper bounds on the scalar charge for $1+2 \lambda_{s}^{2} \varepsilon>0$, which are based on the conjectur on the parameters of solutions $P_{1}=$ $P_{1}\left(Q_{1}^{2}, Q_{2}^{2}\right), P_{2}=P_{2}\left(Q_{1}^{2}, Q_{2}^{2}\right)$. For $\varepsilon=+1$ the lower bound on the gravitational mass is in agreement for $\lambda_{1}=\lambda_{2}$ with that obtained earlier by Gibbons et al. by using certain spinor techniques.

Acknowledgment. The authors acknowledge the support from the Program of target financing of the Ministry of Education and Science of the Republic of Kazakhstan Grant No. F.0755. The paper was also funded by by the Ministry of Education of the Russian Federation (Agreement Number 02.a03.21.0008 of 24 June 2016).

\section{References}

[1] M.E. Abishev, K.A. Boshkayev, V.D. Dzhunushaliev and V.D. Ivashchuk, Dilatonic dyon black hole solutions, Class. Quantum Grav. 32, No. 16, 165010 (2015).

[2] V.D. Ivashchuk and V.N. Melnikov, P-brane black holes for general intersections, Grav. Cosmol. 5, No 4, 313-318 (1999); gr-qc/0002085.

[3] M.E. Abishev, K.A. Boshkayev, V. D. Ivashchuk, A. Malybaev, in preparation.

[4] G.W. Gibbons, D. Kastor, L.A.J. London, P.K. Townsend and J. Traschen, Supersymmetric SelfGravitating Solitons, Nucl. Phys. B 416, 850-880 (1994); arxiv: hep-th/9310118. 